\title{
The effects of AST-120 on chronic kidney disease progression in the United States of America: a post hoc subgroup analysis of randomized controlled trials
}

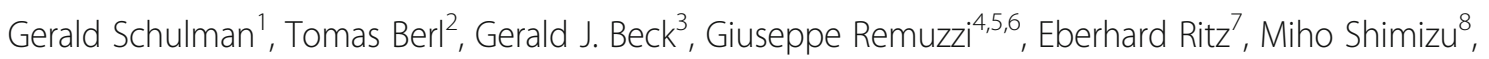
Yuko Shobu ${ }^{9}$ and Mami Kikuchi ${ }^{9^{*}}$

\begin{abstract}
Background: The orally administered spherical carbon adsorbent AST-120 is used on-label in Asian countries to slow renal disease progression in patients with progressive chronic kidney disease (CKD). Recently, two multinational, randomized, double-blind, placebo-controlled, phase 3 trials (Evaluating Prevention of Progression in Chronic Kidney Disease [EPPIC] trials) examined AST-120's efficacy in slowing CKD progression. This study assessed the efficacy of AST-120 in the subgroup of patients from the United States of America (USA) in the EPPIC trials.

Methods: In the EPPIC trials, 2035 patients with moderate to severe CKD were studied, of which 583 were from the USA. The patients were randomly assigned to two groups of equal size that were treated with AST-120 or placebo (9 g/day). The primary end point was a composite of dialysis initiation, kidney transplantation, or serum creatinine doubling.

Results: The Kaplan-Meier curve for the time to achieve the primary end point in the placebo-treated patients from the USA was similar to that projected before the study. The per protocol subgroup analysis of the population from the USA which included patients with compliance rates of $\geq 67 \%$ revealed a significant difference between the treatment groups in the time to achieve the primary end point (Hazard Ratio, 0.74; 95 \% Confidence Interval, 0.56-0.97).

Conclusions: This post hoc subgroup analysis of EPPIC study data suggests that treatment with AST-120 might delay the time to primary end point in CKD patients from the USA. A further randomized controlled trial in progressive CKD patients in the USA is necessary to confirm the beneficial effect of adding AST-120 to standard therapy regimens.
\end{abstract}

Trial registration: ClinicalTrials.gov NCT00500682; NCT00501046.

Keywords: AST-120, Chronic kidney disease, Clinical trial, Spherical carbon adsorbent, Uremic toxin

\section{Background}

Treatment of end-stage renal disease (ESRD) is costly, and the prevalence of ESRD is increasing worldwide [1,2]. The number of ESRD cases per year with diabetes as the primary cause has risen since 1980, with 50,000 ESRD cases in 2009 in the United States of America (USA) [2]. The prevalence of diabetes in ESRD cases in 2012 was $44 \%$ [2]. Diabetic nephropathy occurs in $20-40 \%$ of patients with

\footnotetext{
* Correspondence: mami-k@kureha.co.jp

${ }^{9}$ Kureha Corporation, 3-26-2, Hyakunin-cho, Shinjuku-ku, Tokyo 169-8503, Japan

Full list of author information is available at the end of the article
}

diabetes and is the single leading cause of ESRD [3]. Clearly, preventing or slowing the progression of CKD and diabetes may reduce the cost of ESRD treatment considerably [4-7]. Current guidelines focus on managing factors that can hasten the progression of CKD, such as hypertension and proteinuria [8-10]. Although angiotensin-converting enzyme inhibitors (ACEIs) and angiotensin II receptor blockers (ARBs) slow the progression of CKD, many patients progress to ESRD despite taking these drugs. Because various pathologies contribute to the progression of CKD, additional interventions to decelerate the progression of CKD are needed. 
AST-120 (Kureha Corporation, Tokyo, Japan) is an orally administered spherical carbon adsorbent approved in Japan for delaying the initiation of dialysis and ameliorating uremia symptoms in patients with progressive CKD $[11,12]$. AST-120 has also been approved in Korea, the Philippines, and Taiwan. AST-120 reduces the concentrations of indoxyl sulfate (IS), a uremic toxin that enhances CKD progression, in the systemic circulation. AST-120 lowers IS levels by preventing the absorption of indole, a tryptophan breakdown product and a precursor of IS, from the gastrointestinal tract, which is the presumed mechanism underlying AST-120's effect of slowing the progression of CKD [13].

Recently, two large, multinational, randomized, double-blind, placebo-controlled, phase 3 trials (Evaluating Prevention of Progression in Chronic Kidney Disease [EPPIC-1 and EPPIC-2]) were conducted to assess the effect of AST-120 in adults with CKD treated with standard therapies. The benefit of adding AST-120 to the standard therapy in patients with moderate to severe CKD was not supported by the results of the primary analysis of the intent-to-treat (ITT) population for each EPPIC trial or by the result of the pooled analysis of the two EPPIC trials [14]. We observed that disease progression in the EPPIC trial placebo population was more gradual than that estimated during study planning as reported previously [14]. In contrast, pre-specified subgroup analysis suggested that the population from the USA showed the expected primary event rate. Therefore, in this paper, we compared the end point rate and the disease progression among countries, and we conducted a post hoc subgroup analysis of the patients from the USA who were included in the EPPIC trials (EPPICUSA), hypothesizing that they would be more likely to show a response to AST-120 administration.

\section{Methods}

\section{Study design}

Details of the EPPIC trials have been previously published including the patient flow diagram [14]. The protocols for the EPPIC trials were approved by local ethics committees, and the trials were conducted in accordance with Good Clinical Practice Guidelines of the International Conference on Harmonisation, the Declaration of Helsinki, and the European Union Clinical Trials Directive 2001/20/EC. The EPPIC trials were conducted between July 2007 and February 2012 at 239 international sites to compare the effects of AST-120 with those of placebo on renal outcomes in patients with moderate to severe CKD receiving standard therapy. Patients were randomly assigned to groups of equal size that were treated with $9 \mathrm{~g} /$ day AST-120 or placebo. AST-120 (provided as ten 300-mg capsules 3 times daily) or placebo was administered with meals and at least $1 \mathrm{~h}$ after concomitant medication. The trials were registered on ClinicalTrials.gov (NCT00500682 [EPPIC-1] and NCT00501046 [EPPIC-2]).

\section{Patients}

Eligible patients were adults with moderate to severe CKD (defined as serum creatinine $(\mathrm{sCr})$ at screening of $2.0-5.0$ and $1.5-5.0 \mathrm{mg} / \mathrm{dL}$ for men and women, respectively) who were not expected to require dialysis or renal transplantation within 6 months of trial entry and who were expected to survive for $\geq 1$ year. All patients were required to have proteinuria or progressive deterioration in renal function indicated by either a urinary total protein to urinary creatinine (UP/UCr) ratio $>0.5$ at screening or an $\mathrm{sCr}$ level that had increased by $>10 \%$ at a second evaluation conducted 3 months after screening.

\section{Outcomes}

The primary end point was a triple composite of the time from the date of group assignment to the date of kidney disease progression, as indicated by dialysis initiation, kidney transplantation, or doubling of the $\mathrm{sCr}$ level, whichever occurred first.

\section{Statistical analysis}

The pre-specified statistical methods used for the EPPIC trials' analyses were applied in the same manner for these post hoc subgroup analyses. A pooled population of both EPPIC trials was used for all analyses. The primary end point was analyzed using a stratified Cox proportional hazards regression model with a $95 \%$ confidence interval (CI), and Kaplan-Meier (K-M) methodology was used to calculate the median time from group assignment to renal disease onset. The unstratified Cox regression method was used to analyze the individual components of the composite end point. The covariate adjustments were CKD etiology (diabetic or non-diabetic nephropathy) and baseline $\mathrm{sCr}$ level (greater than or less than $3 \mathrm{mg} / \mathrm{dL}$ ) in all analysis, and region (North America, Latin America, or Europe) was added for the all country primary analysis The unstratified Cox regression method was used to analyze the primary end point within the following baseline subgroups: diabetic nephropathy, $\mathrm{sCr}$ level, CKD stage determined by the estimated glomerular filtration rate (eGFR) level, C-reactive protein (CRP) level, anemia status, age, race, gender, use of ACEI or ARB at baseline, and UP/UCr ratio. Declines in eGFR were calculated for the first 96-week treatment period to elucidate the degree of renal disease progression.

The ITT population included all randomly assigned patients who received at least one dose of the study drug and had at least one post-baseline $\mathrm{sCr}$ measurement. The per protocol (PP) population included all patients in 
Table 1 Primary efficacy end point achievement and disease progression by country (pooled ITT population)

\begin{tabular}{|c|c|c|c|c|c|c|c|c|}
\hline \multirow{2}{*}{\multicolumn{2}{|c|}{ Country }} & \multicolumn{2}{|c|}{ AST-120 } & \multicolumn{2}{|c|}{ Placebo } & \multicolumn{3}{|l|}{ Placebo } \\
\hline & & $\bar{N}$ & $n(\%)$ & $\bar{N}$ & $n(\%)$ & Median Time $^{\text {a }}$ & eGFR decline ${ }^{b}$ & $\overline{\text { eGFR at Dialysis Initiation }{ }^{c}}$ \\
\hline \multicolumn{2}{|l|}{$\overline{A L L}$} & 1000 & $350(35.0)$ & 999 & $360(36.0)$ & 180.1 & $-4.96 \pm 9.50$ & $10.47 \pm 5.28$ \\
\hline \multirow[t]{2}{*}{ NA } & CAN & 61 & $22(36.1)$ & 57 & $19(33.3)$ & ND & $-6.25 \pm 9.56$ & $10.65 \pm 3.28$ \\
\hline & USA & 290 & $120(41.4)$ & 293 & $129(44.0)$ & 135.6 & $-4.66 \pm 11.42$ & $13.95 \pm 5.84$ \\
\hline \multirow[t]{3}{*}{ LA } & ARG & 73 & $20(27.4)$ & 73 & $27(37.0)$ & 135.7 & $-5.17 \pm 7.69$ & $9.15 \pm 2.47$ \\
\hline & BRA & 84 & $26(31.0)$ & 83 & $25(30.1)$ & ND & $-3.13 \pm 6.65$ & $8.82 \pm 3.39$ \\
\hline & MEX & 58 & $21(36.2)$ & 57 & $18(31.6)$ & ND & $-5.13 \pm 8.35$ & $7.45 \pm 3.57$ \\
\hline \multirow[t]{8}{*}{ EU } & CZE & 21 & $6(28.6)$ & 20 & $5(25.0)$ & ND & $-3.52 \pm 3.02$ & $11.91 \pm 1.86$ \\
\hline & DEU & 7 & $2(28.6)$ & 8 & $4(50.0)$ & 119.3 & $-3.49 \pm 14.93$ & $14.77 \pm 3.29$ \\
\hline & ESP & 8 & $3(37.5)$ & 9 & $5(55.6)$ & 71.3 & $-4.20 \pm 4.12$ & $9.08 \pm 1.24$ \\
\hline & FRA & 17 & $5(29.4)$ & 15 & $4(26.7)$ & ND & $-3.93 \pm 7.59$ & $11.24 \pm 2.68$ \\
\hline & ITA & 8 & $4(50.0)$ & 7 & $2(28.6)$ & ND & $-2.60 \pm 2.60$ & $8.18 \pm 1.80$ \\
\hline & $\mathrm{POL}$ & 36 & $18(50.0)$ & 38 & $15(39.5)$ & ND & $-3.50 \pm 6.97$ & $10.00 \pm 3.23$ \\
\hline & RUS & 173 & 57 (32.9) & 174 & $51(29.3)$ & ND & $-4.78 \pm 7.73$ & $7.32 \pm 2.90$ \\
\hline & UKR & 164 & $46(28.0)$ & 165 & $56(33.9)$ & ND & $-7.17 \pm 10.55$ & $5.72 \pm 2.67$ \\
\hline
\end{tabular}

${ }^{a}$ Weeks, ${ }^{b} \mathrm{~mL} / \mathrm{min} / 1.73 \mathrm{~m}^{2} /$ year, ${ }^{\mathrm{c}} \mathrm{mL} / \mathrm{min} / 1.73 \mathrm{~m}^{2}$

ND Not detected

NA North America, LA Latin America, EU Europe, CAN Canada, USA United States of America, ARG Argentina, BRA Brazil, MEX Mexico, CZE Czech Republic, DEU Germany, ESP Spain, FRA France, ITA Italy, POL Poland, RUS Russia, UKR Ukraine

the ITT population without major protocol violations which were: receiving treatment other than that originally assigned, having a treatment compliance rate of $<67 \%$, and/or a treatment period of $<8$ weeks. Inclusion in the PP population was determined during a blinded data review conducted prior to locking of the database and study unblinding. Patients who did not reach the primary end point were censored at the date of last contact. Sensitivity analyses of the primary efficacy end point were performed to evaluate the robustness of the results to censoring patterns.

\section{Results}

The number of patients, primary end point rates, median time to the primary end point, eGFR decline, and eGFR at dialysis initiation by country are summarized in Table 1. While the median time to the primary end point pre-study was estimated to be

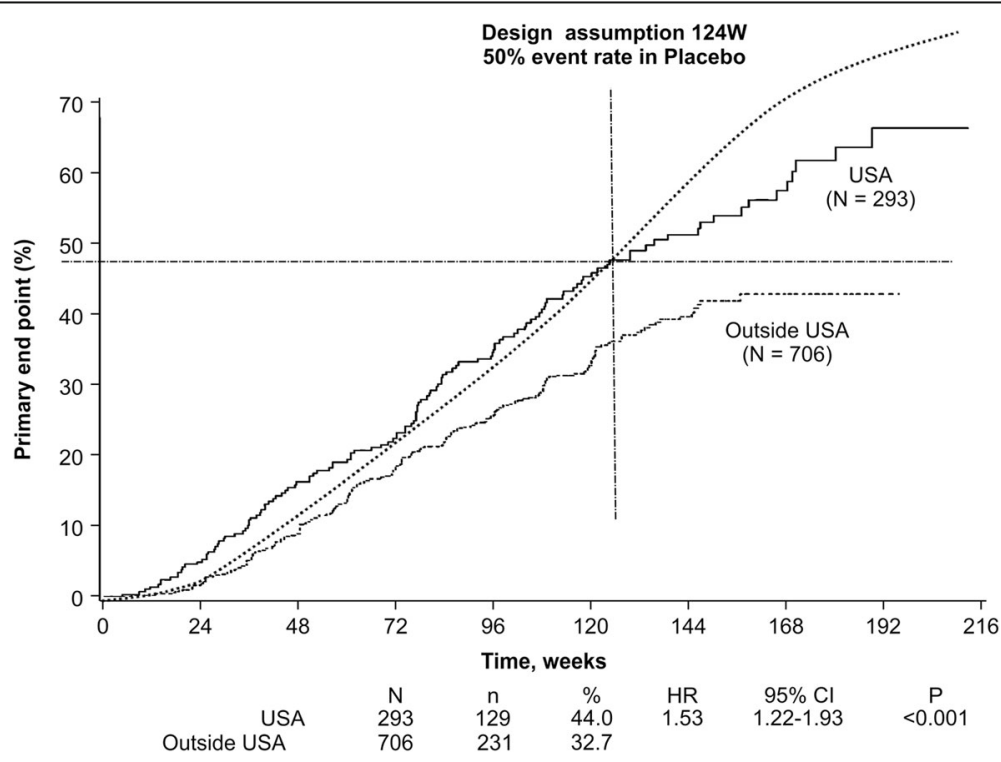

Fig. 1 Kaplan-Meier plots of primary end point achievement in placebo-treated USA and outside USA populations. Cl confidence interval, HR hazard ratio 
124 weeks, the actual time for the entire population was 180.1 weeks. The median time to the primary end point could be calculated only for patients from the USA (135.6), Argentina (135.7), Germany (119.3), and Spain (71.3). Of the countries for which the analysis was possible, the USA had the largest study population (AST-120, $N=290$; placebo, $N=293$ ).

The K-M curve for time to the primary end point for placebo-treated EPPIC-USA patients was similar to the curve estimated during study planning, while the curve for the placebo-treated non-USA patients differed significantly, as shown in Fig. 1. Therefore, we selected the EPPIC-USA population to assess the effect of AST-120 in patients with CKD.

Demographics and baseline clinical characteristics of EPPIC-USA patients are shown in Table 2. Sixty percent of the EPPIC-USA patients were diabetic and $80 \%$ of the EPPIC-USA patients were taking an ACEI or an ARB at baseline data collection. There were no significant differences in demographics and baseline clinical characteristics between the AST-120 and placebo groups. We applied the pre-specified statistical methods used in the EPPIC trials [14] to the EPPIC-USA population.

The results of the primary and secondary analyses, including the sensitivity analyses, are shown in Fig. 2. While there was no significant difference in primary end point achievement between the treatment arms in the EPPIC-USA ITT population (hazard ratio (HR), 0.82; $95 \% \mathrm{CI}, 0.64-1.05 ; P=0.117$ ), treatment with AST-120 reduced the risk of achieving the primary end point in the PP population censored at last contact (HR, 0.74; $95 \% \mathrm{CI}, 0.56-0.97 ; P=0.029)$. K-M curves for the ITT and PP populations are shown in Fig. $3 a$ and $b$, respectively.

In the EPPIC-USA population, the majority of the primary end points were ESRD (defined as dialysis and transplantation), while doubling of $\mathrm{sCr}$ occurred infrequently. In the PP population, treatment with AST-120 reduced the risk of achieving ESRD (HR, 0.70; $95 \% \mathrm{CI}$, 0.53-0.94; $P=0.016$ ) (Fig. 2). The K-M curves for these results are shown in Fig. 3c.

In the subgroup of patients with diabetic nephropathy indicated as the CKD etiology, there was a significant difference in the time to primary end point (HR, 0.70; $95 \% \mathrm{CI}, 0.49-0.99 ; P=0.041)$ and the time to ESRD (HR, 0.65; $95 \%$ CI, 0.45-0.93; $P=0.019$ ) between the AST-120 and placebo groups in the PP population, but there were no significant differences in the time to primary end point in the diabetic nephrology ITT population (HR, 0.77; $95 \% \mathrm{CI}, 0.57-1.06 ; P=0.106$ ) (Fig. 2). The K-M curves for these results are shown in Fig. 3e, f, and $d$, respectively. These results were similar to that of the whole population (Fig. 2, ALL).
Table 2 Demographic and baseline clinical characteristics of the EPPIC-USA population (pooled ITT population)

\begin{tabular}{|c|c|c|c|}
\hline & AST-120 & Placebo & $P$-value \\
\hline & $N=290$ & $N=293$ & \\
\hline Age, years, mean $\pm S D$ & $60.1 \pm 14.3$ & $61.7 \pm 12.3$ & 0.15 \\
\hline \multicolumn{4}{|l|}{ Sex, \% } \\
\hline Male & 64.5 & 70.6 & 0.11 \\
\hline \multicolumn{4}{|l|}{ Race, $\%^{\mathrm{a}}$} \\
\hline White & 66.6 & 60.8 & 0.51 \\
\hline Black or African American & 19.7 & 25.3 & \\
\hline Asian & 6.2 & 5.1 & \\
\hline $\begin{array}{l}\text { Native Hawaiian or other Pacific } \\
\text { Islander }\end{array}$ & 0.7 & 0.7 & \\
\hline American Indian or Alaska Native & 0.0 & 0.3 & \\
\hline Other & 6.9 & 7.8 & \\
\hline \multicolumn{4}{|l|}{ CKD etiology, $\%$} \\
\hline Diabetic nephropathy & 58.6 & 61.8 & 0.44 \\
\hline Type I Diabetes & 6.2 & 3.4 & \\
\hline Type II Diabetes & 52.4 & 58.4 & \\
\hline Non-diabetic nephropathy & 41.4 & 38.2 & \\
\hline Glomerulonephritis & 7.6 & 12.3 & \\
\hline Nephrosclerosis & 16.2 & 15.7 & \\
\hline Other & 17.6 & 10.2 & \\
\hline \multicolumn{4}{|l|}{ Use of ACEl or ARB, \% } \\
\hline Yes & 79.7 & 80.5 & 0.79 \\
\hline Baseline $\mathrm{s} C \mathrm{r}, \mathrm{mg} / \mathrm{dL}$, mean $\pm \mathrm{SD}^{\mathrm{b}}$ & $3.01 \pm 0.84$ & $3.08 \pm 0.80$ & 0.28 \\
\hline $\begin{array}{l}\text { Baseline eGFR, } \mathrm{mL} / \mathrm{min} / 1.73 \mathrm{~m}^{2} \text {, } \\
\text { mean } \pm \mathrm{SD}\end{array}$ & $23.59 \pm 7.79$ & $23.04 \pm 6.92$ & 0.36 \\
\hline \multicolumn{4}{|l|}{ Baseline UP/UCr ratio } \\
\hline N & 288 & 292 & 0.06 \\
\hline Mean \pm SD & $1.94 \pm 1.29$ & $2.15 \pm 1.40$ & \\
\hline \multicolumn{4}{|l|}{ CKD stage, $\%$} \\
\hline Stage $3 a$ & 0.3 & 0.7 & 0.70 \\
\hline Stage $3 b$ & 20.7 & 14.3 & \\
\hline Stage 4 & 64.5 & 74.4 & \\
\hline Stage 5 & 14.5 & 10.6 & \\
\hline \multicolumn{4}{|l|}{ Baseline anemia status, $\%^{c}$} \\
\hline Yes & 76.2 & 79.9 & 0.25 \\
\hline \multicolumn{4}{|l|}{$\mathrm{BMl}, \mathrm{kg} / \mathrm{m}^{2 \mathrm{~d}}$} \\
\hline N & 289 & 293 & 0.20 \\
\hline Mean \pm SD & $31.8 \pm 7.2$ & $32.7 \pm 8.7$ & \\
\hline
\end{tabular}

${ }^{\text {a Race was self-reported }}$

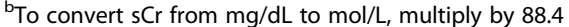

'Anemia was defined as a hemoglobin level $<13.5 \mathrm{~g} / \mathrm{dL}$ (men) or $<12.0 \mathrm{~g} / \mathrm{dL}$ (women)

${ }^{d}$ Body mass index is the weight in kilograms divided by the square of the height in meters 


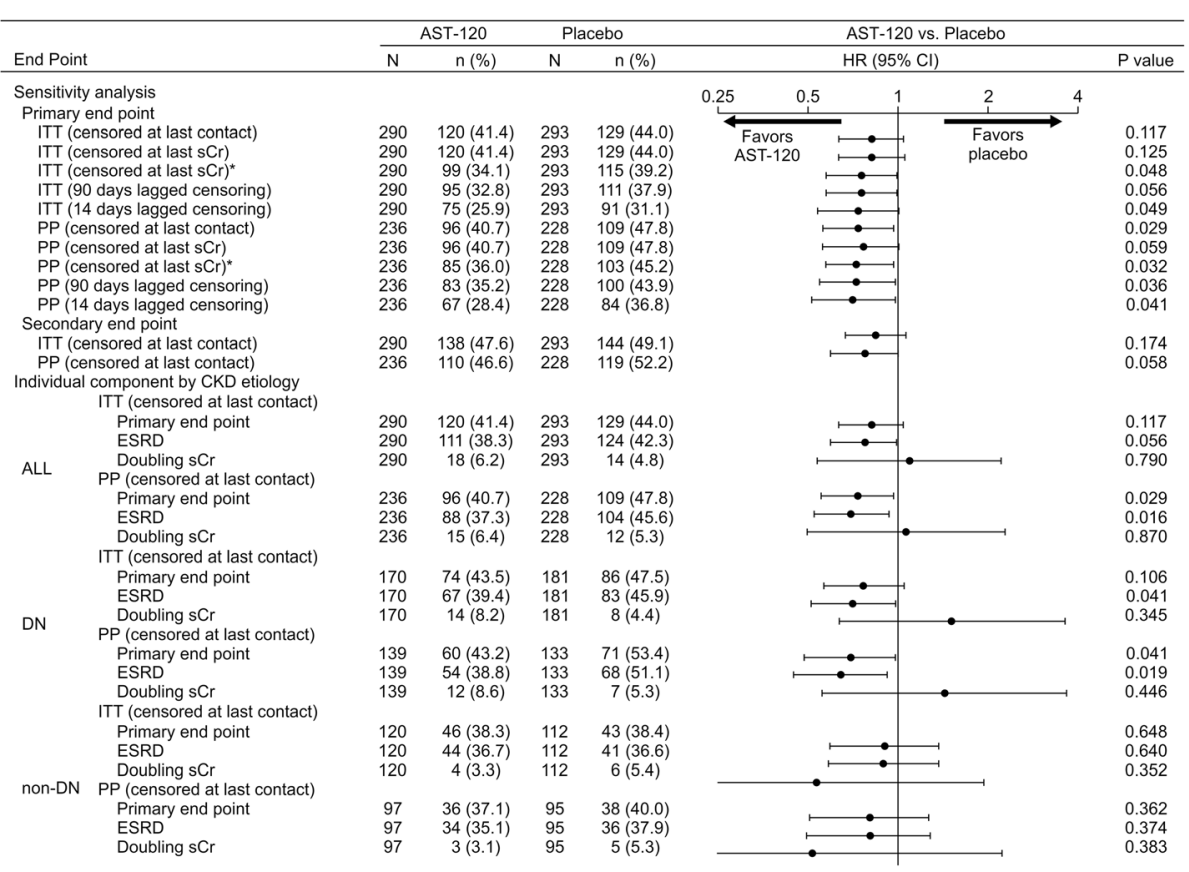

Fig. 2 Achievement of primary and secondary end points in the EPPIC-USA subpopulation. ${ }^{a}$ Sensitivity analyses of the primary efficacy end point were performed to evaluate the robustness of the results to censoring patterns. ${ }^{b}$ First occurrence of dialysis, kidney transplantation, or doubling of $\mathrm{s} C \mathrm{r}$ in the 84 days after the last $\mathrm{s} C r$ assessment or last dose. Patients who did not have an event in this period were censored at the last $\mathrm{sCr}$ assessment. CKD chronic kidney disease, DN diabetic nephropathy, ESRD end stage renal disease, HR hazard ratio, ITT intent-to-treat, PP per protocol, sCr serum creatinine

The results of the subgroup analysis are shown in Fig. 4. Treatment with AST-120 reduced the risk of achieving the primary end point in the white (in race) and ACEI/ARB treatment-receiving patient subgroup. A tendency was observed that AST-120 treatment delays the time to primary end point in patients with $\mathrm{DN}$, with CKD stage 5 and CRP levels $(>3.0 \mathrm{mg} / \mathrm{L})$. No effect was observed in other baseline parameters and demographic characteristics including age and gender.

\section{Discussion}

This post hoc analysis of EPPIC studies in CKD patients from 13 countries demonstrated that the occurrence of primary end point, that is the composite of dialysis initiation, kidney transplantation, and serum creatinine doubling, was different among countries. Particularly, the time to primary end point in the EPPIC-USA placebo population was shorter than that of the placebo population outside the USA $(P<0.001)$. The incidence rates of ESRD continue to become clear based on the enhancement of renal databases in each countries and regions. However, the international difference of CKD progression and time to ESRD of CKD patients have not well discussed and remain unclear.

Recently, the National Kidney Foundation (NKF) and Food and Drug Administration (FDA) published a series of reports showing that eGFR decline associated with
ESRD occurrence [15-18]. However, the value of eGFR decline in the EPPIC-USA placebo population did not differ from that in EPPIC overall population. The time to end point achievement by each country was not related to the observed eGFR decline in the EPPIC population. Thus, the difference in ESRD occurrence among the countries could not be attributed to differences in eGFR decline. Other possible causes may be assumed as following.

First, regional differences in the mean eGFR at dialysis could have contributed to the time to primary end point. Of the countries participating in the EPPIC trials, the USA had the highest mean eGFR at dialysis $(13.53 \pm$ $5.68 \mathrm{~mL} / \mathrm{min} / 1.73 \mathrm{~m}^{2}$ ), which was close to the K/DOQI criterion of $15 \mathrm{~mL} / \mathrm{min} / 1.73 \mathrm{~m}^{2}$ [19]. This observation is consistent with data from the US Renal Data System (USRDS), which shows that $>15 \%$ of patients started dialysis with an eGFR of $\geq 15 \mathrm{~mL} / \mathrm{min} / 1.73 \mathrm{~m}^{2}$ after 2009 [2]. The median eGFR at the start of dialysis was $7.7 \mathrm{~mL} / \mathrm{min} / 1.73 \mathrm{~m}^{2}$ in the 2003 European Renal Association-European Dialysis and Transplant Association Registry [20], notably lower than that in USRDS. Regional differences may account for the observation that the majority of the primary end points were ESRD in the EPPIC-USA population.

Second, the dietary habit and nutritional status are variety among countries and it has been proposed that 


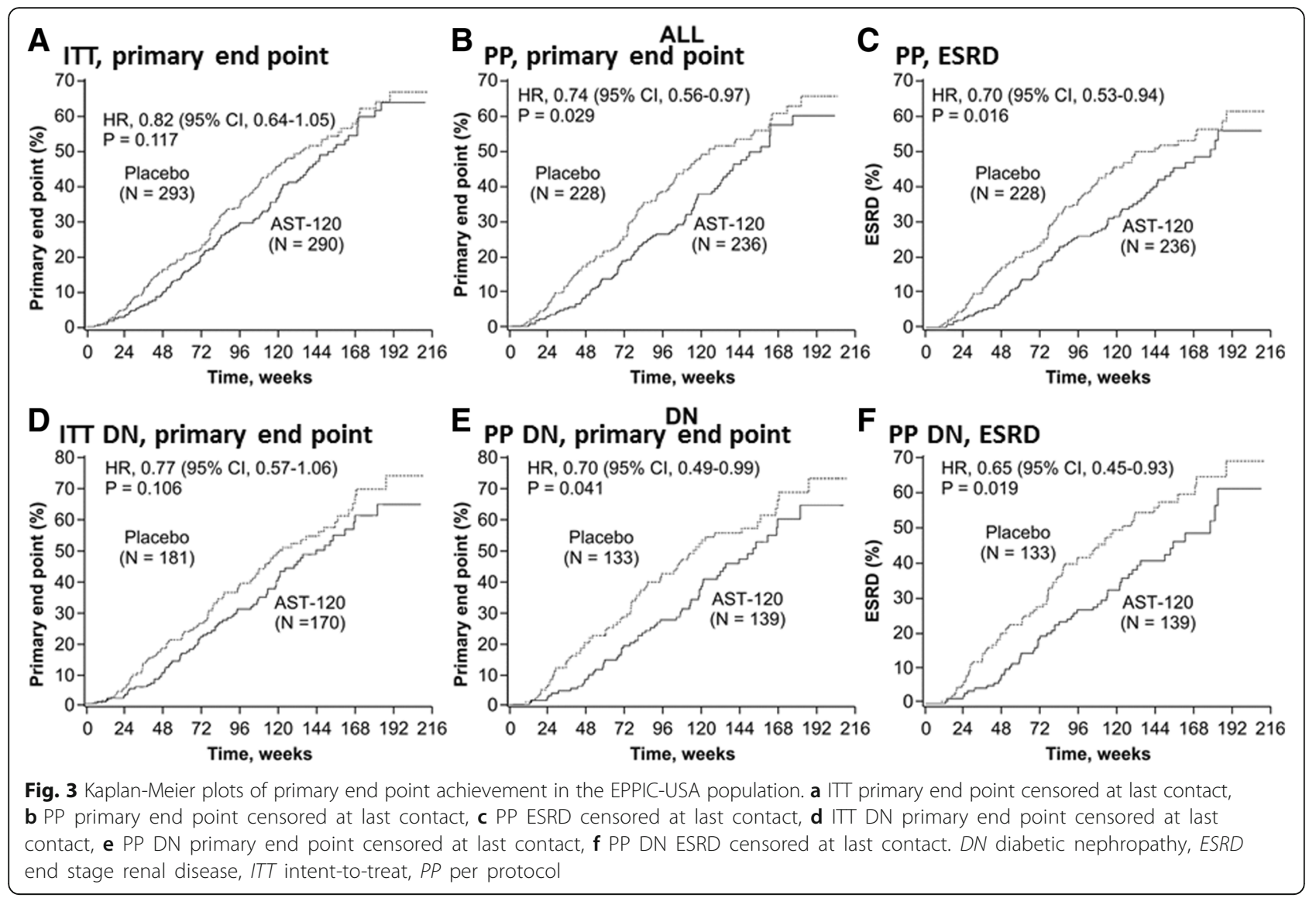

they could affect the CKD progression. The role of nutrition in the progression of CKD is unclear with discrepancies between various studies performed, but some reviews suggested that dietary protein intake [21] and obesity [22] are risk factors for CKD. In EPPIC studies, nutritional information was not collected. However the demographic parameter of patients showed that the mean of body mass index (BMI) and diabetes ratio of USA patients was higher than that of patients outside the USA at baseline $(\mathrm{p}<0.001)$. These could relate to the time to renal events.

The difference in event rates observed between countries may have also been due to the influence of differing healthcare standards and guidelines. Prior to study start, we estimated the primary end point rate for a placebo population based on RENAAL study data; in that study, $45 \%$ of study patients were from the US [23]; we found the median time to primary end point of EPPIC-USA placebo population was similar to the estimated median time of placebo population in the RENNAL study.

A trend for a beneficial effect of AST-120 was observed in the EPPIC-USA PP population comprised of patients who took the study medication as prescribed, had study drug compliance rates $\geq 67 \%$, and were treated for at least 8 weeks. This effect was corroborated by sensitivity analyses that assessed the time lag between treatment cessation and event occurrence. Therefore, good patient compliance highly determines the positive effect of AST-120 in preventing systemic accumulation of uremic toxins, an important factor considering the 30 capsule per day regimen. The difficulty of AST-120 medication may be an issue needing to be solved as relatively large number of capsule should be taken. AST-120 treatment was beneficial in patients with diabetic nephropathy; however, for patients with an etiology other than diabetic nephropathy, there was no difference between the AST-120treated and placebo groups. We speculate that the difference in etiology is responsible for the difference in event rates between the placebo groups with diabetic nephropathy and non-diabetic nephropathy (47.5 vs. $38.4 \%$, respectively). Results from an additional subgroup analysis in ACEI- or ARB-treated patients suggested that the addition of AST-120 to standard therapy produced therapeutic benefits by a distinct mechanism of action that involved lowering levels of uremic toxins such as IS [24].

In addition, examining the use of AST-120 in populations that have a particular rate of disease progression, such as diabetic nephropathy patients or ESRD 


\begin{tabular}{|c|c|c|c|c|c|c|c|}
\hline \multirow{2}{*}{ Subgroup } & \multicolumn{2}{|c|}{ AST-120 } & \multicolumn{2}{|c|}{ Placebo } & \multicolumn{2}{|c|}{ AST-120 vs Placebo } & \multirow[b]{2}{*}{$\mathrm{P}$ value } \\
\hline & $\mathrm{N}$ & $n(\%)$ & $\mathrm{N}$ & $n(\%)$ & \multicolumn{2}{|c|}{$\mathrm{HR}(95 \% \mathrm{Cl})$} & \\
\hline \multirow{2}{*}{\multicolumn{5}{|c|}{ Baseline Diabetic Nephropathy }} & 0.1 & 10 & \\
\hline & & & & $86(47.5)>2>0$ & \multirow{2}{*}{$\begin{array}{l}\text { Favors } \mapsto-1 \\
\text { AST-120 }\end{array}$} & \multirow{2}{*}{$\begin{array}{l}\begin{array}{l}\text { Favors } \\
\text { placebo }\end{array} \\
\end{array}$} & 0.081 \\
\hline No & 120 & $46(38.3)$ & 112 & $43(38.4)$ & & & 0.598 \\
\hline \multicolumn{8}{|l|}{ Baseline sCr Level } \\
\hline & 159 & $45(28.3)$ & 156 & $48(30.8)$ & $\mapsto$ & & 0.578 \\
\hline$>3.0 \mathrm{mg} / \mathrm{dL}$ & 131 & $75(57.3)$ & 137 & $81(59.1)$ & & & 0.114 \\
\hline \multicolumn{8}{|l|}{ At or above age 65} \\
\hline Yes & 118 & $47(39.8)$ & 126 & $55(43.7)$ & & & 0.148 \\
\hline No & 172 & $73(42.4)$ & 167 & $74(44.3)$ & & & 0.178 \\
\hline \multicolumn{8}{|l|}{ Race } \\
\hline White & 193 & $80(41.5)$ & 178 & $82(46.1)$ & & & 0.033 \\
\hline Black or African American & 57 & $24(42.1)$ & 74 & $33(44.6)$ & & & 0.544 \\
\hline Asian & 18 & $4(22.2)$ & 15 & $4(26.7)$ & & & 0.774 \\
\hline Other & 22 & $12(54.5)$ & 26 & $10(38.5)$ & & & 0.471 \\
\hline \multicolumn{8}{|l|}{ Gender } \\
\hline Female & 103 & $44(42.7)$ & 86 & $38(44.2)$ & $\mapsto$ & & 0.177 \\
\hline Male & 187 & $76(40.6)$ & 207 & $91(44.0)$ & & & 0.162 \\
\hline \multicolumn{8}{|l|}{ Use of ACEl or ARB at baseline } \\
\hline Yes & 231 & $89(38.5)$ & 236 & 106(44.9) & & & 0.024 \\
\hline No & 59 & $31(52.5)$ & 57 & $23(40.4)$ & & & 0.541 \\
\hline \multicolumn{8}{|c|}{ CKD stage determined by eGFR level } \\
\hline Stage 3 & 61 & $12(19.7)$ & 44 & $5(11.4)$ & & & \\
\hline Stage 4 & 187 & $80(42.8)$ & 218 & $101(46.3)$ & & & 0.102 \\
\hline Stage 5 & 42 & $28(66.7)$ & 31 & $23(74.2)$ & 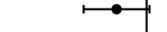 & & 0.075 \\
\hline \multicolumn{8}{|l|}{ CRP lev } \\
\hline$<1.0 \mathrm{mg} / \mathrm{L}$ & 74 & $31(41.9)$ & 72 & $32(44.4)$ & & & 0.626 \\
\hline $1.0-3.0 \mathrm{mg} / \mathrm{L}$ & 80 & $33(41.3)$ & 91 & $40(44.0)$ & & & 0.533 \\
\hline$>3.0 \mathrm{mg} / \mathrm{L}$ & 133 & $56(42.1)$ & 128 & $57(44.5)$ & $\mapsto$ & & 0.074 \\
\hline \multicolumn{8}{|l|}{ Anemia } \\
\hline & 221 & $106(48.0)$ & 234 & $111(47.4)$ & $\mapsto$ & & 0.211 \\
\hline No & 69 & $14(20.3)$ & 58 & $18(31.0)$ & & & 0.101 \\
\hline \multicolumn{8}{|l|}{ UP/UCr at baseline } \\
\hline$<2.0$ & 182 & $57(31.3)$ & 162 & $51(31.5)$ & & & 0.468 \\
\hline$>=2.0$ & 106 & $63(59.4)$ & 130 & $78(60.0)$ & - & & 0.217 \\
\hline
\end{tabular}

Fig. 4 Achievement of primary end points in the EPPIC-USA subgroups. ACEl angiotensin converting enzyme inhibitor, ARB angiotensin receptor blocker, CKD chronic kidney disease, CRP C-reactive protein, eGFR estimated glomerular filtration rate, sCr serum creatinine, UP/UCr Urinary total protein to urinary creatinine ratio

patients with residual renal function, may be useful. Atoh et al. reported a strong correlation between IS serum levels and eGFRs [25]. The EUTox group reported that IS serum levels were increased significantly and progressively as patients progressed through the stages of CKD, including predialysis and hemodialysis patients [26].

\section{Conclusions}

This post hoc subgroup analysis of EPPIC study data suggests that treatment with AST-120 might delay the time to primary end point in CKD patients from the USA. The major limitations of this analysis are its post hoc nature and its use of one subgroup population. The conclusions of this study are hypothesis-generating only, and the $p$-values should not be interpreted by the usual standards regarding a Type-1 error threshold of 0.05 . According to our findings, a randomized controlled trial in progressive CKD patients in the USA is necessary to confirm the beneficial effect of adding AST-120 to standard therapy regimens.

\section{Abbreviations}

ACEIs: Angiotensin-converting enzyme inhibitors; ARBs: Angiotensin II receptor blockers; Cl: Confidence interval; CKD: Chronic kidney disease; CRP: C-reactive protein; DN: Diabetic nephropathy; eGFR: Estimated glomerular filtration rate; EPPIC: Evaluating Prevention of Progression in Chronic Kidney Disease trials; EPPIC-USA: Patients from the USA who were included in the EPPIC trials; ESRD: End-stage renal disease; HR: Hazard ratio; IS: Indoxyl sulfate; ITT: Intent-to-treat; K-M: Kaplan-Meier; PP: Per protocol; sCr: Serum creatinine; UP/UCr: Urinary total protein to urinary creatinine ratio; USA: United States of America; USRDS: US Renal Data System

\section{Acknowledgement}

The EPPIC trials were sponsored by Mitsubishi Tanabe Pharma Corporation and Kureha Corporation, Tokyo, Japan. The baseline characteristics of the patients enrolled in the EPPIC trials were presented at the National Kidney Foundation 2012 Spring Clinical Meeting, held May 9-13, 2012, in

Washington, DC. Results from the EPPIC trials were presented at the 2012 Annual Meeting of the American Society of Nephrology, held October 30-November 4, 2012, in San Diego, California.

Details of the EPPIC trials including the EPPIC committee members and the persons participated in the EPPIC trials have been previously published [14].

\section{Authors' contributions}

Author contributions are listed below: GS, TB, GJB, GR, and ER conceived and designed the study, revised the manuscript critically for important intellectual content, approved the final draft of the manuscript for publication, and agreed to be accountable for all aspects of the work in ensuring that questions related to the accuracy or integrity of any part of the work are appropriately investigated and resolved. MS conceived and designed the study and analyzed and interpreted the data, drafted and 
revised the manuscript critically for important intellectual content, approved the final draft of the manuscript for publication, and agreed to be accountable for all aspects of the work in ensuring that questions related to the accuracy or integrity of any part of the work are appropriately investigated and resolved. YS and MK collected data and analyzed and interpreted data, drafted or revised the manuscript critically for important intellectual content, approved the final draft of the manuscript for publication, and agreed to be accountable for all aspects of the work in ensuring that questions related to the accuracy or integrity of any part of the work are appropriately investigated and resolved. All authors read and approved the final manuscript.

\section{Competing interest}

Gerald Schulman reports having received consulting fees or honoraria, payment for lectures, including service on speakers bureaus, and support for travel to meetings for the study or other purposes from Kureha Corporation and Mitsubishi Tanabe Pharma Corporation. Tomas Berl reports having received fees for participation in review activities, such as data monitoring boards, statistical analysis, and end point committees, from Kureha Corporation and Mitsubishi Tanabe Pharma Corporation; and consulting fees from Sanofi, fees for expert testimony from AstraZeneca, and payment for lectures, including service on speakers bureaus, from Otsuka. Gerald Beck reports having received consulting fees from Kureha Corporation and Mitsubishi Tanabe Pharma Corporation as a steering committee member for EPPIC-1 and -2, and consulting fees or honoraria for participation in other review activities, such as data and safety monitoring boards. Giuseppe Remuzzi reports having received consulting fees or honoraria from Kureha Corporation and Mitsubishi Tanabe Pharma Corporation, and his institution has received payment for consultancy work from Alexion Pharmaceuticals, AstraZeneca, Pharmanet, and Reata Pharmaceuticals. Eberhard Ritz reports receiving payment for lectures, including service on speakers bureaus, from AbbVie, Amgen, Daiichi Sankyo, and Medice; and receiving consulting fees from Kureha Corporation and Mitsubishi Tanabe Pharma Corporation as a steering committee member for EPPIC-1 and -2. Miho Shimizu reports employment with Mitsubishi Tanabe Pharma Corporation. Yuko Shobu reports employment with Kureha Corporation. Mami Kikuchi reports employment with Kureha Corporation.

\section{Ethics approval and consent to participate}

The protocols for the EPPIC trials were approved by local ethics committees, and the trials were conducted in accordance with Good Clinical Practice Guidelines of the International Conference on Harmonisation, the Declaration of Helsinki, and the European Union Clinical Trials Directive 2001/20/EC. The trials were registered on ClinicalTrials.gov (NCT00500682 [EPPIC-1] and NCT00501046 [EPPIC-2]). Informed consent was obtained from all patients.

\section{Author details}

${ }^{1}$ Vanderbilt University School of Medicine, Nashville, TN, USA. ${ }^{2}$ University of Colorado Health Sciences Center, Denver, CO, USA. ${ }^{3}$ Cleveland Clinic Foundation, Cleveland, OH, USA. ${ }^{4}$ Unit of Nephrology and Dialysis, Azienda Ospedaliera Papa Giovanni XXIII, Bergamo, Italy. ${ }^{5}$ IRCCS Istituto di Ricerche Farmacologiche Mario Negri, Bergamo, Italy. ${ }^{6}$ Department of Biomedical and Clinical Sciences, University of Milan, Milan, Italy. ${ }^{7}$ University of Heidelberg, Heidelberg, Germany. ${ }^{8}$ Mitsubishi Tanabe Pharma Corporation, Tokyo, Japan. ${ }^{9}$ Kureha Corporation, 3-26-2, Hyakunin-cho, Shinjuku-ku, Tokyo 169-8503, Japan.

\section{Received: 21 January 2016 Accepted: 26 September 2016} Published online: 30 September 2016

\section{References}

1. ERA-EDTA Registry. ERA-EDTA Registry Annual Report 2012. Academic Medical Center, Department of Medical Informatics, Amsterdam, The Netherlands. 2014.

2. United States Renal Data System, 2014 Annual Data Report. Epidemiology of kidney disease in the United States. National Institutes of Health, National Institute of Diabetes and Digestive and Kidney Diseases, Bethesda, MD. 2014.

3. American Diabetes Association. Standards of medical care in diabetes 2014. Diabetes Care. 2014;37 Suppl 1:S14-80.
4. Brown JB, Pedula KL, Bakst AW. The progressive cost of complications in type 2 diabetes mellitus. Arch Intern Med. 1999;159:1873-80.

5. Pelletier EM, Smith PJ, Boye KS, et al. Direct medical costs for type 2 diabetes mellitus complications in the US commercial payer setting: a resource for economic research. Appl Health Econ Health Policy. 2008;6:103-12.

6. Herman WH, Shahinfar S, Carides GW, et al. Losartan reduces the costs of end-stage renal disease: the RENAAL study economic evaluation. Diabetes Care. 2003;26:683-7.

7. Hayashino Y, Fukuhara S, Akizawa T, et al. CAP-KD study group. Costeffectiveness of administering oral adsorbent AST-120 to patients with diabetes and advance-stage chronic kidney disease. Diabetes Res Clin Pract. 2010;90:154-9.

8. National Kidney Foundation. $\mathrm{KDOQ}$ clinical practice guideline for diabetes and CKD: 2012 update. Am J Kidney Dis. 2012;60:850-86.

9. National Kidney Foundation. KDOQI clinical practice guidelines on hypertension and antihypertensive agents in chronic kidney disease. Am J Kidney Dis. 2004;43 Suppl 1:S1-S290

10. Andrassy KM. National Kidney Foundation: comments on 'KDIGO 2012 clinical practice guideline for the evaluation and management of chronic kidney disease'. Kidney Int. 2013;84:622-3.

11. Akizawa T, Koide K, Koshikawa S. Effects of Kremezin on patients with chronic renal failure results of a nationwide clinical study. Kidney Dial. 1998; 45:373-88 [Japanese].

12. Schulman G, Vanholder R, Niwa T. AST-120 for the management of progression of chronic kidney disease. Int J Nephrol Renovasc Dis. 2014; 7:49-56.

13. Niwa T, Yazawa $T$, Ise $M$, et al. Inhibitory effect of oral sorbent on accumulation of albumin-bound indoxyl sulfate in serum of experimental uremic rats. Nephron. 1991;57:84-8.

14. Schulman G, Berl T, Beck GJ, et al. Randomized Placebo-Controlled EPPIC Trials of AST-120 in CKD. J Am Soc Nephrol. 2015;26:1732-46.

15. Coresh J, Turin TC, Matsushita K, et al. Decline in estimated glomerular filtration rate and subsequent risk of end-stage renal disease and mortality. JAMA. 2014;311:2518-31.

16. Lambers Heerspink HJ, Weldegiorgis M, Inker LA, et al. Estimated GFR decline as a surrogate end point for kidney failure: a post hoc analysis from the Reduction of End Points in Non-Insulin-Dependent Diabetes With the Angiotensin II Antagonist Losartan (RENAAL) study and Irbesartan Diabetic Nephropathy Trial (IDNT). Am J Kidney Dis. 2014;63:244-50.

17. Inker LA, Lambers Heerspink HJ, Mondal H, et al. GFR decline as an alternative end point to kidney failure in clinical trials: a meta-analysis of treatment effects from 37 randomized trials. Am J Kidney Dis. 2014;64:848-59.

18. Lambers Heerspink HJ, Tighiouart H, Sang Y, et al. GFR decline and subsequent risk of established kidney outcomes: a meta-analysis of 37 randomized controlled trials. Am J Kidney Dis. 2014;64:860-6.

19. National Kidney Foundation. KDOQI clinical practice guidelines and clinical practice recommendations for 2006 updates: hemodialysis adequacy, peritoneal dialysis adequacy and vascular access. Am J Kidney Dis. 2006;48:S1-S322.

20. Stel VS, Tomson C, Ansell D, et al. Level of renal function in patients starting dialysis: and ERA-EDTA Registry study. Nephrol Dial Transplant. 2010;25: 3315-25.

21. Fouque D, Laville M. Low protein diets for chronic kidney disease in non diabetic adults. Cochrane Database Syst Rev. 2009;3:CD001892.

22. Ladhani M, Craig JC, Irving M, et al. Obesity and the risk of cardiovascular and all-cause mortality in chronic kidney disease: a systematic review and meta-analysis. Nephrol Dial Transplant. 2016. doi:10.1093/ndt/gfw075.

23. Keane WF, Brenner BM, Zeeuw D, et al. The risk of developing end-stage renal disease in patients with type 2 diabetes and nephropathy: The RENAAL Study. Kidney Int. 2003;53:1499-507.

24. Schulman G, Agarwal R, Acharya M, et al. A multicenter, randomized, double-blind, placebo-controlled, dose-ranging study of AST-120 (Kremezin) in patients with moderate to severe CKD. Am J Kidney Dis. 2006;47:565-77.

25. Atoh $\mathrm{K}$, Itoh $\mathrm{H}$, Haneda M. Serum indoxyl sulfate levels in patients with diabetic nephropathy: relation to renal function. Diabetes Res Clin Pract. 2009;83:220-6.

26. Barreto FC, Barreto DV, Liabeuf S, et al. European Uremic Toxin Work Group (EUTOX): serum indoxyl sulfate is associated with vascular disease and mortality in chronic kidney disease patients. Clin J Am Soc Nephrol. 2009:4:1551-8. 